\title{
Visual Symptoms in Parkinson's Disease
}

\author{
R. A. Armstrong \\ Department of Vision Sciences, Aston University, Birmingham B4 7ET, UK \\ Correspondence should be addressed to R. A. Armstrong, r.a.armstrong@aston.ac.uk
}

Received 26 October 2010; Revised 21 February 2011; Accepted 24 March 2011

Academic Editor: Irena Rektorova

Copyright ( $) 2011$ R. A. Armstrong. This is an open access article distributed under the Creative Commons Attribution License, which permits unrestricted use, distribution, and reproduction in any medium, provided the original work is properly cited.

Parkinson's disease (PD) is a common disorder of middle-aged and elderly people in which degeneration of the extrapyramidal motor system causes significant movement problems. In some patients, however, there are additional disturbances in sensory systems including loss of the sense of smell and auditory and/or visual problems. This paper is a general overview of the visual problems likely to be encountered in PD. Changes in vision in PD may result from alterations in visual acuity, contrast sensitivity, colour discrimination, pupil reactivity, eye movements, motion perception, visual field sensitivity, and visual processing speeds. Slower visual processing speeds can also lead to a decline in visual perception especially for rapidly changing visual stimuli. In addition, there may be disturbances of visuospatial orientation, facial recognition problems, and chronic visual hallucinations. Some of the treatments used in PD may also have adverse ocular reactions. The pattern electroretinogram (PERG) is useful in evaluating retinal dopamine mechanisms and in monitoring dopamine therapies in PD. If visual problems are present, they can have an important effect on the quality of life of the patient, which can be improved by accurate diagnosis and where possible, correction of such defects.

\section{Introduction}

Parkinson's disease (PD) is a common neurodegenerative disorder affecting middle aged and elderly people. It is a disease characterised by deficiency of dopamine in areas of the midbrain causing a variety of movement problems such as akinesia, rigidity, and tremor. Despite the emphasis on motor function in $\mathrm{PD}$, nonmotor symptoms may also play a significant role in determining the general quality of life of the patient. Hence, the symptoms of PD can include depression, apathy, sleep problems, cognitive impairment, dementia, and autonomic, gastrointestinal, and sensory problems [1]. Sensory problems may include visual loss, loss of smell, auditory problems, and "restless legs" syndrome (RLS). Visual signs and symptoms of PD may include defects in eye movement, pupillary function, and in more complex visual tasks involving the ability to judge distance or the shape of an object $[2,3]$. The symptoms of PD can be treated successfully using drug therapy or surgery, and these treatments may also have visual side effects. Hence, this paper provides a general overview of (1) the visual signs and symptoms of PD, (2) the areas of the eye and brain which may be affected by the pathology of $\mathrm{PD}$, and (3) the adverse ocular reactions to treatment.

\section{Visual Symptoms in Parkinson's Disease}

$\mathrm{PD}$ is associated with a variety of visual problems and these are summarised in Table 1.

2.1. Visual Acuity. PD patients often complain of poor vision especially as the disease progresses resulting, in part, from poor visual acuity [4], low contrast acuity being especially affected $[5,6]$. Impaired visual acuity also appears to be a risk factor for the development of chronic hallucinations in PD [7]. Poor visual acuity may be caused by lack of dopamine in the retina, abnormal eye movements, or poor blinking and is only marginally improved by drug therapy [6].

2.2. Colour Vision. Vision has been reported to be blurred in PD to coloured stimuli [8] with reduced colour fusion times [9] which indicate the accuracy of perception of monochromatic contours. A progressive deterioration of colour 
TABLE 1: Visual signs and symptoms of Parkinson's disease (PD).

\begin{tabular}{|c|c|c|}
\hline Ocular aspect & Change in PD & References \\
\hline \multirow{2}{*}{ Visual acuity } & \multirow{2}{*}{ Poor, especially at low contrast } & {$[6]$} \\
\hline & & [4] \\
\hline \multirow{3}{*}{ Colour vision } & Vision blurred for coloured stimuli & [8] \\
\hline & Shortened colour fusion time & [9] \\
\hline & Progressive deterioration & {$[10]$} \\
\hline \multirow{2}{*}{ Visual fields } & Increase in glaucomatous visual field defects & [13] \\
\hline & Side effects of surgery & [14] \\
\hline \multirow{3}{*}{ Saccadic eye movement } & Reaction time and max. velocity of horizontal gaze slower & [15] \\
\hline & Hypometria & [16] \\
\hline & Amplitude increased after cued saccades & [17] \\
\hline \multirow{3}{*}{ Smooth pursuit movement } & Affected early in disease process & {$[20]$} \\
\hline & Superimposed saccades & [15] \\
\hline & Reduction in response magnitude & [15] \\
\hline Optokinetic Nystagmus & Abnormal in some patients & [15] \\
\hline \multirow{2}{*}{ Convergence } & \multirow{2}{*}{ Impaired, associated with large exophoria, diplopia } & [21] \\
\hline & & [22] \\
\hline Blink frequency & Reduced, causing abnormal tear film, dry eye and reduced vision & [23] \\
\hline Blink reflex & Habituation not observed & [24] \\
\hline Pupil diameter & Larger after light adaptation with anisocoria & [26] \\
\hline Light reflex & Longer latency & \\
\hline Constriction time & Increased & [26] \\
\hline Contraction amplitude & Reduced & [23] \\
\hline Contrast sensitivity (CS) & Abnormal in some cases, intermediate to high frequencies & [28] \\
\hline \multirow{2}{*}{ Temporal processing } & Impaired ability to track rapid fluctuations & [32] \\
\hline & Duration perception affected & [33] \\
\hline \multirow[t]{2}{*}{ Flash ERG } & Reduced amplitude of "b" wave & [35] \\
\hline & Reduced amplitudes. & {$[35]$} \\
\hline \multirow[t]{2}{*}{ PERG } & Specific defect at medium SF & \\
\hline & Delayed P50 & [36] \\
\hline \multirow[t]{2}{*}{ Cortical VEP } & Delayed P100 & [45] \\
\hline & & [46] \\
\hline \multirow{2}{*}{ Chromatic VEP } & Increased latency and reduced & {$[44]$} \\
\hline & Amplitude (esp. blue-yellow) & \\
\hline \multirow[t]{2}{*}{ ERP } & \multirow[t]{2}{*}{ Abnormal. Delayed reaction times } & [42] \\
\hline & & [41] \\
\hline \multirow{2}{*}{ Visuo-spatial } & Difficulty in judging verticals, & [48] \\
\hline & position of body parts, and in route-walking tasks & [49] \\
\hline Orientation and motion discrimination & Impaired & [50] \\
\hline Facial perception & Impaired ability to perceive and imagine emotional faces & [51] \\
\hline Visual hallucinations & Chronic in $30-60 \%$ of treated cases & [54] \\
\hline
\end{tabular}

Abbreviations: ERG: Electroretinogram, ERP: event-related potentials, PERG: Pattern electroretinogram, SF: Spatial frequency, VEP: Visual evoked potentials.

discrimination is also evident and is often associated with impairments of higher motor function [10]. Using the Farnsworth-Munsell 100-hue test, however, colour visual discrimination does not appear to be consistently impaired in the early stages of PD [11].
2.3. Visual Fields. There have been few studies of visual field defects in patients with PD [12]. Retrospective analysis of ophthalmic charts from PD patients, however, using a cupto-disc ratio of 0.8 or greater to define glaucoma, revealed glaucomatous visual field defects in approximately $24 \%$ of 
patients suggesting there may be an increased rate of glaucoma in PD [13]. In addition, intraocular pressure (IOP) was slightly higher in PD patients with glaucoma compared with glaucoma patients without PD (mean 18.9 compared with 16.0). Of eight PD patients with glaucoma, five were considered to have low tension glaucoma. In one study, visual fields were investigated in patients undergoing posterior pallidotomy, a procedure which risks damaging structures such as the optic tract [14]. Of 40 such patients, three had visual field defects likely to be attributable to the surgery, namely, contralateral superior quadrantanopia, associated in two patients with small paracentral scotomas.

2.4. Saccadic and Smooth Pursuit Eye Movements. Assessment of oculomotor function in PD can be made clinically or by using electro-oculography (EOG). EOG responses are often normal in PD patients when the eyes are in the primary position or when resting. Abnormal saccadic and smooth pursuit eye movements, however, have been reported in about $75 \%$ of patients [15]. Both reaction times and the maximum saccadic velocity of horizontal gaze are slower in PD [15]. Saccadic eye movements may exhibit hypometria, that is, "under reaching of task" [16] while smooth pursuit movements may be interrupted by small saccades [15]. In addition, the amplitude of saccadic eye movements are increased in normal subjects when there is a change from externally cued saccades to self-paced saccades and this effect is often greater in PD [17]. In a study in which the delay of remembered (imagined) saccades was gradually increased in untreated PD patients, there was a marked hypometria of saccadic gain at all delays suggesting a dysfunction of the striatocollicular inhibitory pathways in PD attributable to dopamine deficiency in the basal ganglia [18]. In a further experiment, spatial working memory was studied in relation to eye movements [19]. A sequence of four targets was memorised by the patient and then the eyes were moved to fixate the targets in their correct order. In PD, several discrete saccadic eye movements of reduced amplitude were necessary before reaching the final eye position, and the patients also exhibited an increasing proportion of errors in remembering the target sequence. The results suggested that memory representation was disrupted early in the development of PD.

EOG recordings have been made before and after apomorphine treatment in patients with early-stage disease and have confirmed that smooth pursuit movements are affected during the initial stages of the disease [20]. In addition, patients with PD often have difficulty in sustaining repetitive actions and hence, smooth pursuit movements exhibit a reduction in response magnitude and a progressive decline of response with stimulus repetition.

2.5. Nystagmus and Convergence. Abnormal optokinetic nystagmus "train nystagmus" [15] and convergence [21] have been reported in PD patients. Further abnormalities that have been observed include "jerkiness", "cogwheeling", and limitation of eye movement. Vertical eye movements are often more impaired than horizontal movements. Convergence can be associated with relatively large exophoria (outward deviation of the eye), and the result is often diplopia (double vision) [22].

2.6. Blink Reflex. Patients with PD exhibit a reduced frequency of blinking leading to a staring appearance [23]. Reduced blink rate can cause an abnormal tear film, dry eye, and reduced vision. A characteristic ocular sign may be the blink reflex, elicited by a light tap above the bridge of the nose, successive taps in normal individuals producing less and less response as the reflex habituates [24]. In PD, the blink reflex may not disappear on repeated tapping. In addition, blink duration may be increased in PD reflecting the loss of dopamine neurons [25].

2.7. Pupil Reactivity. Significantly larger pupil diameters, with anisocoria (unequal pupil sizes) after light adaptation, have been reported in PD [26], no differences being observed after dark adaptation. In addition, longer light reflex latencies and constriction times have been observed while contraction amplitudes may be reduced [23]. These results suggest that there is an autonomic imbalance in PD patients involving the parasympathetic system.

2.8. Psychophysics. Contrast sensitivity (CS) is affected in a proportion of $\mathrm{PD}$ patients especially at the high or intermediate frequencies [27-29]. In some individuals, a substantial decrease in CS can be demonstrated as the disease progresses and could be a contributory cause of poor vision in PD. Abnormalities in CS are likely to be related to dopamine dysfunction and are often orientation specific suggesting cortical involvement [30]. L-dopa therapy generally improves CS performance close to that of healthy control patients without any neurological dysfunction. In addition, apomorphine significantly improves achromatic spatial CS at all spatial frequencies but appears to have minimal effects on colour vision [31].

There may be decreased sensitivity to temporally changing stimuli in PD which have been well demonstrated by studies of the auditory system. Hence, in psychophysical tests assessing auditory processing, bilateral subthalamic nucleus stimulation caused dysfunction in ability to track rapid fluctuations in sound intensity [32]. In addition, in motor tasks involving finger tapping, a PD group were impaired both in the motor task itself and in assessing duration implicating the basal ganglia and thalamocortical connections in timing [33]. Subsequently, the substantia nigra, an important site of PD pathology, was shown to be involved in temporal processing involving motor and perceptual tasks [34]. Problems in the visual perception of rapidly moving stimuli are likely to cause problems in tracking fast moving targets.

2.9. Electrophysiology. Significant changes in the electroretinogram (ERG) have been found in PD. Studies show that the amplitude of the ERG " $\mathrm{b}$ " wave may be reduced in PD patients under a variety of light conditions [35]. Since 
the amplitude of the " $b$ " wave may be a diagnostic indicator of the functioning of the inner nuclear layer, the reduction may reflect defects in visual processing involving dopamine neurons. In addition, the amplitude of the pattern ERG (PERG) to a checkerboard stimulus is decreased [35] and the latency of the P50 component delayed [36] in PD patients. Subsequent studies have suggested that retinal dopamine depletion may result in attenuated ERG responses to peak stimuli [37]. Two dopamine sensitive pathways have been postulated: (1) involving the D1 receptors which primarily affect the "surround" organisation of ganglion cells with large centres and (2) the D2 postsynaptic receptors which contribute to the "centre" response amplification of ganglion cells with smaller centres. In addition, steady-state pattern PERG to sinusoidal gratings was studied over a range of spatial frequencies [38]. Aging affected responses at all spatial frequencies but the pattern of age-related loss was different in PD. In PD, there was a specific deficit at medium spatial frequencies accompanied by a distorted PERG spatial frequency response function. PERG is also sensitive to dopamine manipulation in the monkey retina [39]. In a further experiment involving the use of the selective D2 blocker l-sulpiride, treatment affected the PERG to a sinusoidal vertical grating presented at four spatial frequencies [39]. The data suggested that dopamine is involved in retinal processing in primates and that the D2 receptor is necessary for spatial-temporal tuning of pattern vision. Subsequently it was shown that the two dopamine receptors play different roles in retinal function and therefore in the different visual alterations in PD [40]. Hence, PERG is useful in evaluating retinal dopamine mechanisms and in monitoring dopamine therapies in PD.

Event-related potentials (ERP) employing various "oddball" tasks have been used to study the sensory and cognitive processing in PD. Abnormal ERP responses in PD often correlate with worsening Wechsler and motor dysfunctional scale scores [41]. In a study of the P300 response, believed to reflect orientation, attention, stimulus evaluation, and memory, reductions in reaction time were actually less in PD than in other "parkinsonian syndromes" such as progressive supranuclear palsy (PSP) and corticobasal degeneration (CBD) [42].

Evoked responses to coloured stimuli are also affected in PD [43] supporting the hypothesis that dopamine modulates the retinal colour system. In idiopathic PD, for example, amplitude is decreased and latency increased for all chromatic stimuli and especially for those using blue-yellow (BY) horizontal gratings [44], and this test may be a simple tool for distinguishing different parkinsonian syndromes. Increased latency of the visually evoked potential (VEP) P100 peak to a checkerboard stimulus has been reported in a proportion of patients suggesting a delay in visual processing at one or more stages of the visual system [45-47].

2.10. Complex Visual Functions. There are prominent deficits in $\mathrm{PD}$ involving neuropsychological tests requiring selfmotivation and a demanding response from the patient [1]. $\mathrm{PD}$ patients may exhibit a variety of deficits in visuo-spatial orientation $[48,49]$ including difficulty in judging verticals and the position of body parts, and in carrying out a routewalking task. Visuo-spatial working memory appears to be selectively impaired early in PD which probably reflects degeneration of the basal ganglia, the dorsal visual stream, and the frontal-prefrontal cortex [1]. Patients may also have problems with memory tasks involving spatial orientation. PD patients often show an impairment of orientation and motion discrimination [50] suggesting that the visual pathway beyond the retina may be affected since these tasks are most likely to involve the visual cortex. In addition, impairments in the ability to perceive and imagine faces have been reported in PD [51]. Medicated and unmedicated patients exhibit facial recognition problems but these deficits are most frequently present in the untreated group [52]. Normal subjects contract their facial muscles while imaging faces, a process which is often impaired in PD patients. In a problem solving task involving arranging coloured balls in pockets on a computer screen, PD patients made more errors on the task than controls and also did not show any dissociation in the amount of time fixating the two halves of the display [53]. The results suggested difficulties in encoding and/or maintaining current goals during problem solving in PD.

2.11. Visual Hallucinations. Visual hallucinations are a chronic complication of PD $[54,55]$ and especially in patients treated with L-dopa and dopamine agonists. In a large study of PD patients, hallucinations occurred in the previous three months in $40 \%$ of patients examined. Hallucinations were visual in $22 \%$ and auditory in approximately $10 \%$ of patients [55]. Patients with minor hallucinations had higher depression scores than those without. Three factors were the best predictors of hallucinations, namely, severe cognitive defects, daytime somnolence, and longer duration of disease. Hallucinations in PD are often complex with flickering lights, and illusionary misconceptions often preceding the most common manifestation, namely, stereotypical colourful images. Visual hallucinations may involve a disturbance in the regulation of the gating and filtering of external perception and internally generated visual images. Risk factors for hallucinations in PD patients include poor primary vision and reduced activity of the primary visual cortex (area V1).

\section{Pathological Changes Affecting the Visual System}

3.1. Eye Pathology. Few pathological changes have been reported in the eye in PD with the exception of the retina [56]. However, the maximum contraction ability of the iris muscle measured in vitro is greater in PD than in controls suggesting that the muscle may have acquired adaptive sensitivity changes [57].

Dopamine is an important neurotransmitter in the retina and is present in amacrine cells and along the inner border of the inner nuclear layer [58] (Figure 1). In addition, dopamine may be accumulated by interplexiform cells [59]. Two types of amacrine cells appear to be involved. Type 


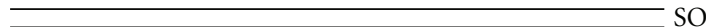

GC

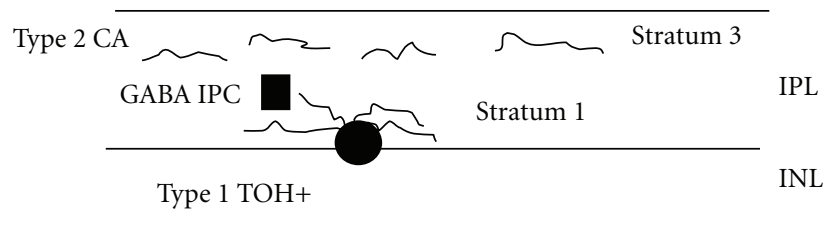

OPL

ONL

PR

PE

Figure 1: The layers of the retina (PE: pigment epithelium, PR: visual receptors, ONL: outer nuclear layer, OPL: outer plexiform layer, INL: internal nuclear layer, IPL: internal plexiform layer, GC: ganglion cell layer, and SO: Stratum opticum). Dopamine neurons (TOH+: Tyrosine hydroxylase positive neurons, Type 1 cells and Type 2 amacrine cells) are primarily concentrated in the INL and dopamine positive neurites (Type 1 in stratum 1 and type 2 ramify above stratum 1) in the IPL. Type 1 cells may synapse onto GABA interpelexiform cells (IPCs). Some dopamine activities may also be observed in the ganglion cell layer.

1 cells send ascending processes to the outer plexiform layer where they synapse with $\gamma$-aminobutyric acid (GABA) interplexiform cells in stratum 1 , whereas type 2 cells have their dendrites stratifying above those of the type 1 cells of the inner plexiform layer (Figure 1). Dopamine may be involved in the organisation of the ganglion cell and bipolar cell receptive fields and appears to modulate the physical activity of the photoreceptors [60]. In addition, dopamine is involved in the coupling of the horizontal and amacrine lateral system [61].

Pathological changes which have been observed in the PD retina include cell losses, which often affect the peripheral segments of the retina more severely and reductions in retinal dopamine [62]. In addition, the thickness of the circumpapillary retinal nerve fibre layer was studied using optical coherence tomography (OCT) [63]. The inferior quadrant layer, and especially the inferior temporal region, was significantly thinner in PD than in controls. In normal subjects, the foveola contains no dopamine neurons, innervation being achieved by processes originating in the avascular zone. In $\mathrm{PD}$, swelling and loss of these processes has been observed. These observations are consistent with the ERG data and support the hypothesis that at least some of the cortical VEP changes could be retinal in origin.

3.2. Brain Pathology. The surviving neurons of the substantia nigra and cerebral cortex often contain inclusions called

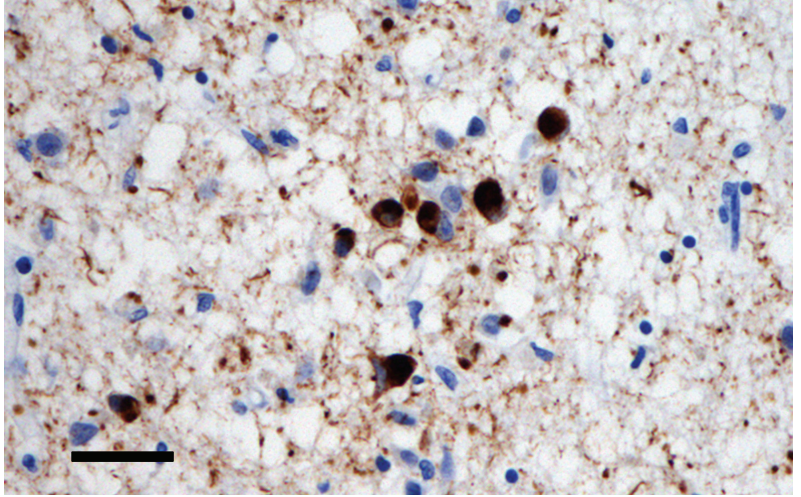

FIGURE 2: Cells from the cerebral cortex showing the presence of Lewy bodies ( $\alpha$-synuclein immunohistochemistry, haematoxylin stain, bar $=25 \mu \mathrm{m})$.

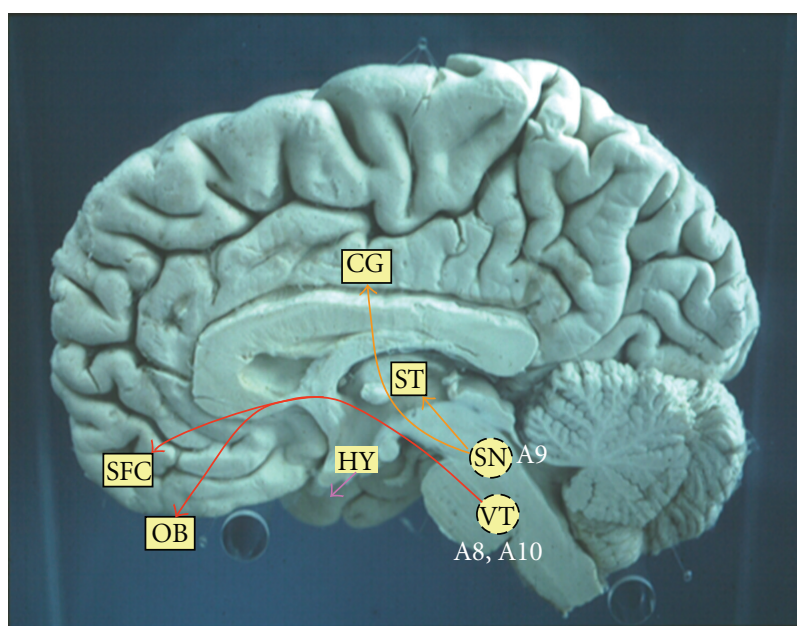

Figure 3: The dopamine projections of the central nervous system (OB: Olfactory bulb, SFC: superior frontal cortex, CG: Cingulate gyrus, ST: Striatum, HY: Hypothalamus, VT: Ventral tegmentum, SN: Substantia nigra).

Lewy bodies (LB) (Figure 2). LB are found in the cytoplasm of the cell and may be derived from cytoskeletal filaments. Recent research suggests that LB differ significantly from other neurofibrillary pathologies in neurodegenerative disease, for example, the neurofibrillary tangles (NFT) found in $\mathrm{AD}$ [64], in that they contain abnormal aggregates of the protein $\alpha$-synuclein [65]. $\alpha$-Synuclein is a small presynaptic protein and the entire molecule undergoes a conformational change to result in the insoluble protein that forms a major component of the LB.

There are two major dopamine pathways in the brain (Figure 3). First, there is the striatonigral pathway from the substantia nigra (cell group A9) to the cortex and striatum. Second, a major pathway originates in the ventral tegmentum (cell groups A8, A10) and projects to the amygdala, septum, nucleus accumbens, olfactory tubercle, and frontal cortex. There are also dopamine pathways within the hypothalamus. Hence, within the brain significant dopamine activity is limited to the frontal and limbic areas of 
TABLE 2: Adverse ocular reactions to treatment for Parkinson's disease.

\begin{tabular}{|c|c|c|}
\hline Treatment & Examples & Ocular side effects \\
\hline Anticholinergi & Benzhexol, Diphenydramine & $\begin{array}{l}\text { Mydriasis, photophobia, dry eyes, decreased accommodation, anisocoria, blurred } \\
\text { vision, anterior angle closure }\end{array}$ \\
\hline \multirow{3}{*}{ Dopamine agonists } & Bromocriptine & May exacerbate visual hallucinations \\
\hline & Pramipexole & May exacerbate visual hallucinations \\
\hline & Ropinirole & May exacerbate visual hallucinations \\
\hline L-dopa & L-dopa/carbidopa & Mydriasis, miosis, blepharospasm eyelid ptosis, may prolong latency of saccades \\
\hline MAO inhibitors & Selegiline & May cause loss of visual acuity and blurred vision \\
\hline Antiviral & Amantadine & Mydriasis, superficial keratitis, reduced accommodation, hallucinations \\
\hline Antidepressant & Imipramine & Mydriasis, cycloplegia, dry eyes, ocular muscle paresis, nystagmus \\
\hline
\end{tabular}

the cerebral cortex with significantly less activity in the visual cortex [62]. Cerebral metabolic rates for glucose, however, are reduced by up to $23 \%$ in the primary visual cortex of PD patients [66]. Reductions in dopamine levels in the basal ganglia and frontal cortex may also deplete levels in the superior colliculus and thus could be a factor in the production of defective saccades [16].

Within the cerebral cortex, functional MRI (fMRI) and EEG studies have both revealed the essential role of the occipital cortex in producing saccadic eye movements while PET studies have revealed occipital hypometabolism in these areas in PD. In addition, in a study using "event-related" fMRI and which utilised a visual attention/motor inhibition task, during motor inhibition there was activation of the prefrontal cortex and basal ganglia [67]. In addition, there was a reduced and less coherent haemodynamic response in the occipital cortex. Hence, specific functional changes involving the frontostriatal network and temporal-occipital cortex were present in the early stages of PD. PD patients with damage to the medial temporal lobe perform the poorest in all explicit memory tasks and memory problems are often apparent in this group in early stage disease [68].

In subcortical regions, areas of the basal ganglia appear to be the most affected by the developing pathology. Within the basal ganglia, the substantia nigra pars reticulata, the subthalamic nucleus, and the caudate nucleus are all involved in saccadic eye movements [69]. There is, however, an overlap in the anatomical pathways involved in saccadic and smooth pursuit movements which may explain why both are affected in PD. Dopamine also has a peripheral role in sympathetic ganglia, visceral ganglia, and in all artery walls. Hence, reductions in dopamine in some of these areas could be a factor contributing to eye movement problems and defects in pupil reactivity.

\section{Adverse Ocular Reactions to Treatment}

There are several drugs given alone or in combination used to treat PD (Table 2). Most act on the brain either by reducing cholinergic activity or by encouraging dopamine activity in the basal ganglia [70].
Anticholinergic drugs such as benzhexol and diphenydramine act to decrease acetylcholine levels, the effect of which is enhanced by the lack of dopamine. Benzhexol may have a significant mydriatic effect and therefore, should not be given to patients with anterior angle closure and should be used with caution in those with a narrow anterior chamber angle. Prolonged exposure to this drug in a few patients may cause an angle closure of gradual onset but without acute symptoms. Optometrists may be the only health practitioners aware of this risk, that is, it is always important to assess anterior chamber depth in PD patients. In addition, photophobia and decreased accommodation can occur resulting in blurred vision [71].

Dopamine agonists such as bromocriptine, pramipexole, and ropinirole enhance the effect of dopamine by directly stimulating dopamine receptors. Pramipexole and ropinirole are often indicated as a treatment for early stage PD and RLS. These drugs may cause less motor complications and dyskinesia than L-dopa but are often given in combination with the latter. Use of dopamine agonists may exacerbate visual hallucinations in PD.

L-dopa is a precursor of dopamine and can penetrate the blood-brain barrier more successfully than dopamine itself. It is often given with a peripheral decarboxylase inhibitor, for example, carbidopa, to reduce the breakdown of L-dopa outside the brain. Mydriasis may occur at first, and this may be followed by miosis. Lid ptosis and blepharospasm have been reported in a few patients [72]. In addition, L-dopa may prolong the latency of saccades [73].

Monoamine oxidase B (MAO-B) inhibitors, such as seliginine, slow down the breakdown of dopamine at the synapse. Patients treated with MAO-B inhibitors and multiple ergotamine-derived dopamine agonists may exhibit blurring of vision [74].

The antiviral drug amantadine appears to have a beneficial effect on many of the symptoms of the disease. A few adverse reactions have been reported including a superficial keratitis, mydriasis and reduced accommodation while in some patients visual hallucinations may occur [75]. By contrast, imipramine has antidepressant and anticholinergic properties and acts by inhibiting the reuptake of dopamine. Ocular side effects include mydriasis, cycloplegia, dry eyes, nystagmus, and the paresis of ocular muscles. 


\section{Discussion and Conclusions}

Patients who have been diagnosed as having PD may develop a range of visual problems during the course of the disease. Hence, changes in vision in PD may result from alterations in visual acuity, contrast sensitivity, colour discrimination, pupil reactivity, eye movements, motion perception, visual field sensitivity, and visual processing speeds. Slower visual processing speeds can also lead to a decline in visual perception especially for rapidly changing visual stimuli. In addition, there may be disturbances of visuo-spatial orientation, facial recognition problems, and chronic visual hallucinations. Some of the treatments used in PD may also have adverse ocular reactions. Visual deficits in PD are important in influencing overall motor function [10], are a risk factor for developing hallucinations [7] and are important in influencing general quality of life [7]. Hence, identifying and correcting the visual problems as far as possible can significantly benefit a PD patient.

Clinical examination of the patient by eye practitioners requires sensitivity to both the physical disability and mental state of the patient and the problems involved have been described in detail by Naylor [70]. Some of the visual problems may be adverse reactions to treatment. Side effects may occur relatively rapidly at the beginning of, or after a change, in drug treatment, but can also occur after a long latent period. It is important that those symptoms due to adverse reactions are distinguished from those due to the disease process itself. If ocular side effects are identified and become severe, then it is essential that these are monitored and the patient referred back to their physician for further clinical assessment.

\section{References}

[1] A. Antal, F. Bandini, S. Kéri, and I. Bodis-Wollner, "Visuocognitive dysfunctions in Parkinson's disease," Clinical Neuroscience, vol. 5, supplement 2, pp. 147-152, 1998.

[2] R. A. Armstrong, "Parkinson's disease and the eye," Ophthalmic and Physiological Optics, vol. 17, no. 2, pp. S9-S16, 1997.

[3] R. A. Armstrong, "Visual signs and symptoms of Parkinson's disease," Clinical and Experimental Optometry, vol. 91, no. 2, pp. 129-138, 2008.

[4] M. X. Repka, M. C. Claro, D. N. Loupe, and S. G. Reich, "Ocular motility in Parkinson's disease," Journal of Pediatric Ophthalmology and Strabismus, vol. 33, no. 3, pp. 144-147, 1996.

[5] R. D. Jones and I. M. Donaldson, "Fractionation of visuoperceptual dysfunction in Parkinson's disease," Journal of the Neurological Sciences, vol. 131, no. 1, pp. 43-50, 1995.

[6] R. D. Jones, I. M. Donaldson, and P. L. Timmings, "Impairment of high-contrast visual acuity in Parkinson's disease," Movement Disorders, vol. 7, no. 3, pp. 232-238, 1992.

[7] H. Matsui, F. Udaka, A. Tamura et al., "Impaired visual acuity as a risk factor for visual hallucinations in Parkinson's disease," Journal of Geriatric Psychiatry and Neurology, vol. 19, no. 1, pp. 36-40, 2006.

[8] M. J. Price, R. G. Feldman, D. Adelberg, and H. Kayne, "Abnormalities in color vision and contrast sensitivity in Parkinson's disease," Neurology, vol. 42, no. 4, pp. 887-890, 1992.
[9] T. Buttner, W. Kuhn, P. Klotz et al., "Disturbance of colour perception in Parkinson's disease," Journal of Neural Transmission, vol. 6, no. 1, pp. 11-15, 1993.

[10] N. J. Diederich, R. Raman, S. Leurgans, and C. G. Goetz, "Progressive worsening of spatial and chromatic processing deficits in Parkinson disease," Archives of Neurology, vol. 59, no. 8, pp. 1249-1252, 2002.

[11] O. Veselá, E. Růžička, R. Jech et al., "Colour discrimination impairment is not a reliable early marker of Parkinson's disease," Journal of Neurology, vol. 248, no. 11, pp. 975-978, 2001.

[12] S. Ture, I. Inci, and M. Gedizlioglu, "Abnormalities of contrast sensitivity, visual fields and visual evoked potentials in Parkinson's disease and effect of dopaminergic treatment," Journal of Neurology, vol. 254, supplement 3, p. 93, 2007.

[13] A. U. Bayer, O. N. Keller, F. Ferrari, and K. P. Maag, "Association of glaucoma with neurodegenerative diseases with apoptotic cell death: Alzheimer's disease and Parkinson's disease," American Journal of Ophthalmology, vol. 133, no. 1, pp. 135137, 2002.

[14] V. Biousse, N. J. Newman, C. Carroll et al., "Visual fields in patients with posterior GPi pallidotomy," Neurology, vol. 50, no. 1, pp. 258-265, 1998.

[15] H. Shibasaki, S. Tsuji, and Y. Kuroiwa, "Oculomotor abnormalities in Parkinson's disease," Archives of Neurology, vol. 36, no. 6, pp. 360-364, 1979.

[16] T. Crawford, S. Goodrich, L. Henderson, and C. Kennard, "Predictive responses in Parkinson's disease: manual keypresses and saccadic eye movements to regular stimulus events," Journal of Neurology Neurosurgery and Psychiatry, vol. 52, no. 9, pp. 1033-1042, 1989.

[17] C. Winograd-Gurvich, N. Georgiou-Karistianis, P. B. Fitzgerald, L. Millist, and O. B. White, "Self-paced saccades and saccades to oddball targets in Parkinson's disease," Brain Research, vol. 1106, no. 1, pp. 134-141, 2006.

[18] S. Shaunak, E. O’Sullivan, S. Blunt et al., "Remembered saccades with variable delay in Parkinson's disease," Movement Disorders, vol. 14, no. 1, pp. 80-86, 1999.

[19] T. L. Hodgson, W. H. Dittrich, L. Henderson, and C. Kennard, "Eye movements and spatial working memory in Parkinson's disease," Neuropsychologia, vol. 37, no. 8, pp. 927-938, 1999.

[20] M. Bares, M. Brazdil, P. Kanovsky et al., "The effect of apomorphine administration on smooth pursuit ocular movements in early Parkinson's disease," Parkinsonism \& Related Disorders, vol. 9, pp. 139-144, 2003.

[21] M. S. Corin, T. S. Elizan, and M. B. Bender, "Oculomotor function in patients with Parkinson's disease," Journal of the Neurological Sciences, vol. 15, no. 3, pp. 251-265, 1972.

[22] F. E. Lepore, "Parkinson's disease and diplopia," NeuroOphthalmology, vol. 30, no. 2-3, pp. 37-40, 2006.

[23] V. Biousse, B. C. Skibell, R. L. Watts, D. N. Loupe, C. DrewsBotsch, and N. J. Newman, "Ophthalmologic features of Parkinson's disease,” Neurology, vol. 62, no. 2, pp. 177-180, 2004.

[24] H. G. Garland, "Refresher course for general practitioners. Parkinsonism," British Medical Journal, vol. 1, pp. 153-155, 1952.

[25] K. R. Peshori, E. J. Schicatano, R. Gopalaswamy, E. Sahay, and C. Evinger, "Aging of the trigeminal blink system," Experimental Brain Research, vol. 136, no. 1, pp. 351-363, 2001.

[26] G. Micieli, C. Tassorelli, E. Martignoni et al., "Disordered pupil reactivity in Parkinson's disease," Clinical Autonomic Research, vol. 1, no. 1, pp. 55-58, 1991. 
[27] C. Bulens, J. D. Meerwaldt, G. J. Van Der Wildt, and J. B. P. Van Deursen, "Effect of levodopa treatment on contrast sensitivity in Parkinson's disease," Annals of Neurology, vol. 22, no. 3, pp. 365-369, 1987.

[28] J. T. Hutton, J. L. Morris, and J. W. Elias, "Levodopa improves spatial contrast sensitivity in Parkinson's disease," Archives of Neurology, vol. 50, no. 7, pp. 721-724, 1993.

[29] I. Bodis-Wollner, M. S. Marx, S. Mitra, P. Bobak, L. Mylin, and M. Yahr, "Visual dysfunction in Parkinson's disease. Loss in spatiotemporal contrast sensitivity," Brain, vol. 110, no. 6, pp. 1675-1698, 1987.

[30] R. L. Rodnitzky, "Visual dysfunction in Parkinson's disease," Clinical Neuroscience, vol. 5, no. 2, pp. 102-106, 1998.

[31] T. H. Büttner, T. H. Müller, and W. Kuhn, "Effects of apomorphine on visual functions in Parkinson's disease," Journal of Neural Transmission, vol. 107, no. 1, pp. 87-94, 2000.

[32] D. Guehl, P. Burbaud, C. Lorenzi et al., "Auditory temporal processing in Parkinson's disease," Neuropsychologia, vol. 46, no. 9, pp. 2326-2335, 2008.

[33] D. L. Harrington, K. Y. Haaland, and N. Hermanowicz, "Temporal processing in the basal ganglia," Neuropsychology, vol. 12, no. 1, pp. 3-12, 1998.

[34] C. R. Jones and M. Jahanshahi, "The substantia nigra, the basal ganglia, dopamine and temporal processing," Journal of Neural Transmission, no. 73, pp. 161-171, 2009.

[35] I. Gottlob, E. Schneider, W. Heider, and W. Skrandies, "Alteration of visual evoked potentials and electroretinograms in Parkinson's disease," Electroencephalography \& Clinical Neurophysiology, vol. 66, pp. 349-357, 1987.

[36] A. Peppe, P. Stanzione, F. Pierelli, D. De Angelis, M. Pierantozzi, and G. Bernardi, "Visual alterations in de novo Parkinson's disease: pattern electroretinogram latencies are more delayed and more reversible by levodopa than are visual evoked potentials," Neurology, vol. 45, pp. 1144-1148, 1995.

[37] I. Bodis-Wollner and A. Tzelepi, "The push-pull action of dopamine on spatial tuning of the monkey retina: the effects of dopaminergic deficiency and selective D and D receptor ligands on the pattern electroretinogram," Vision Research, vol. 38, no. 10, pp. 1479-1487, 1998.

[38] M. Tagliati, I. Bodis-Wollner, and M. D. Yahr, "The pattern electroretinogram in Parkinson's disease reveals lack of retinal spatial tuning," Electroencephalography and Clinical Neurophysiology, vol. 100, no. 1, pp. 1-11, 1996.

[39] M. Tagliati, I. Bodis-Wollner, I. Kovanecz, and P. Stanzione, "Spatial frequency tuning of the monkey pattern ERG depends on D2 receptor-linked action of dopamine," Vision Research, vol. 34, no. 16, pp. 2051-2057, 1994.

[40] P. Stanzione, I. Bodis-Wollner, M. Pierantozzi et al., "A mixed D1 and D2 antagonist does not replay pattern electroretinogram alterations observed with a selective D2 antagonist in normal humans: relationship with Parkinson's disease pattern electroretinogram alterations," Clinical Neurophysiology, vol. 110, no. 1, pp. 82-85, 1999.

[41] M. Li, Y. Kuroiwa, L. Wang et al., "Early sensory information processes are enhanced on visual oddball and S1-S2 tasks in Parkinson's disease: a visual event-related potentials study," Parkinsonism and Related Disorders, vol. 9, no. 6, pp. 329-340, 2003.

[42] L. Wang, Y. Kuroiwa, T. Kamitani et al., "Visual event-related potentials in progressive supranuclear palsy, corticobasal degeneration, striatonigral degeneration, and Parkinson's disease," Journal of Neurology, vol. 247, no. 5, pp. 356-363, 2000.
[43] L. Barbato, S. Rinalduzzi, M. Laurenti, S. Ruggieri, and N. Accornero, "Color VEPs in Parkinson's disease," Electroencephalography and Clinical Neurophysiology, vol. 92, no. 2, pp. 169-172, 1994.

[44] F. Sartucci, G. Orlandi, U. Bonuccelli et al., "Chromatic pattern-reversal electroretinograms (ChPERGs) are spared in multiple system atrophy compared with Parkinson's disease," Neurological Sciences, vol. 26, no. 6, pp. 395-401, 2006.

[45] I. Bodis-Wollner and M. D. Yahr, "Measurements of visual evoked potentials in Parkinson's disease," Brain, vol. 101, no. 4, pp. 661-671, 1978.

[46] M. Onofrj and I. Bosid-Wollner, "Dopaminergic deficiency causes delayed visual evoked potentials in rats," Annals of Neurology, vol. 11, no. 5, pp. 484-490, 1982.

[47] I. Bodis-Wollner, M. D. Yahr, L. Mylin, and J. Thornton, "Dopaminergic deficiency and delayed visual evoked potentials in humans," Annals of Neurology, vol. 11, no. 5, pp. 478483, 1982.

[48] B. E. Levin, M. M. Llabre, S. Reisman et al., "Visuospatial impairment in Parkinson's disease," Neurology, vol. 41, no. 3, pp. 365-369, 1991.

[49] S. Davidsdottir, A. Cronin-Golomb, and A. Lee, "Visual and spatial symptoms in Parkinson's disease," Vision Research, vol. 45, no. 10, pp. 1285-1296, 2005.

[50] G. L. Trick, B. Kaskie, and S. B. Steinman, "Visual impairment in Parkinson's disease: deficits in orientation and motion discrimination," Optometry and Vision Science, vol. 71, no. 4, pp. 242-245, 1994.

[51] P. J. Lang, "Presidential address, 1978. A bio-informational theory of emotional imagery," Psychophysiology, vol. 16, no. 6, pp. 495-512, 1979.

[52] R. Sprengelmeyer, A. W. Young, K. Mahn et al., "Facial expression recognition in people with medicated and unmedicated Parkinson's disease," Neuropsychologia, vol. 41, no. 8, pp. 1047-1057, 2003.

[53] T. L. Hodgson, B. Tiesman, A. M. Owen, and C. Kennard, "Abnormal gaze strategies during problem solving in Parkinson's disease," Neuropsychologia, vol. 40, no. 4, pp. 411-422, 2002.

[54] N. J. Diederich, C. G. Goetz, and G. T. Stebbins, "Repeated visual hallucinations in Parkinson's disease as disturbed external/internal perceptions: focused review and a new integrative model," Movement Disorders, vol. 20, no. 2, pp. 130-140, 2005.

[55] G. Fénelon, F. Mahieux, R. Huon, and M. Ziégler, "Hallucinations in Parkinson's disease. Prevalence, phenomenology and risk factors," Brain, vol. 123, no. 4, pp. 733-745, 2000.

[56] L. A. Hunt, A. A. Sadun, and C. J. Bassi, "Review of the visual system in Parkinson's disease," Optometry and Vision Science, vol. 72, no. 2, pp. 92-99, 1995.

[57] P. N. Patil and T. F. Mauger, "Cholinergic sensitivity of irides from donors with various pathological conditions and lens implants," Naunyn-Schmiedeberg's Archives of Pharmacology, vol. 346, no. 6, pp. 620-628, 1992.

[58] J. E. Dowling, B. Ehinger, and W. L. Hedden, "The interplexiform cell: a new type of retinal neuron," Investigative Ophthalmology, vol. 15, no. 11, pp. 916-926, 1976.

[59] J. M. Frederick, M. E. Rayborn, and A. M. Laties, "Dopaminergic neurons in the human retina," Journal of Comparative Neurology, vol. 210, no. 1, pp. 65-79, 1982.

[60] G. Masson, D. Mestre, and O. Blin, "Dopaminergic modulation of visual sensitivity in man," Fundamental and Clinical Pharmacology, vol. 7, no. 8, pp. 449-463, 1993.

[61] M. B. A. Djamgoz, M. W. Hankins, J. Hirano, and S. N. Archer, "Neurobiology of retinal dopamine in relation to degenerative 
states of the tissue," Vision Research, vol. 37, no. 24, pp. 35093529, 1997.

[62] J. Nguyen-Legros, C. Harnois, T. Di Paolo, and A. Simon, "The retinal dopamine system in Parkinson's disease," Clinical Vision Sciences, vol. 8, no. 1, pp. 1-12, 1993.

[63] R. Inzelberg, J. A. Ramirez, P. Nisipeanu, and A. Ophir, "Retinal nerve fiber layer thinning in Parkinson disease," Vision Research, vol. 44, no. 24, pp. 2793-2797, 2004.

[64] R. A. Armstrong and A. B. Syed, "Alzheimer's disease and the eye," Ophthalmic and Physiological Optics, vol. 16, no. 1, pp. S2-S8, 1996.

[65] M. G. Spillantini, R. Anthony Crowther, R. Jakes, N. J. Cairns, P. L. Lantos, and M. Goedert, "Filamentous $\alpha$-synuclein inclusions link multiple system atrophy with Parkinson's disease and dementia with Lewy bodies," Neuroscience Letters, vol. 251, no. 3, pp. 205-208, 1998.

[66] J. L. Eberling, B. C. Richardson, B. C. Reed, N. Wolfe, and W. J. Jagust, "Cortical glucose metabolism in Parkinson's disease without dementia," Neurobiology of Aging, vol. 15, no. 3, pp. 329-335, 1994.

[67] F. Baglio, V. Blasi, A. Falini et al., "Functional brain changes in early Parkinson's disease during motor response and motor inhibition," Neurobiology of Aging, vol. 32, no. 1, pp. 115-124, 2009.

[68] B. Rieger and H. J. Markowitsch, "Implicit and explicit mnestic performance of patients with prefrontal, medial temporal, and basal ganglia damage," Neurology Psychiatry and Brain Research, vol. 4, no. 2, pp. 53-74, 1996.

[69] M. A. Basso, J. J. Pokorny, and P. Liu, "Activity of substantia nigra pars reticulata neurons during smooth pursuit eye movements in monkeys," European Journal of Neuroscience, vol. 22, no. 2, pp. 448-464, 2005.

[70] R. J. Naylor, "The ocular effects of parkinsonism and its treatment," Optometry in Practice, vol. 6, pp. 19-31, 2005.

[71] Z. Friedman and E. Neumann, "Benzhexol-induced blindness in Parkinson's disease," British Medical Journal, vol. 1, no. 800, p. 605, 1972.

[72] A. S. Spiers, D. B. Calne, and P. M. Fayers, "Miosis during Ldopa therapy," British Medical Journal, vol. 2, no. 7, pp. 639640, 1970.

[73] A. W. Michell, Z. Xu, D. Fritz et al., "Saccadic latency distributions in Parkinson's disease and the effects of L-dopa," Experimental Brain Research, vol. 174, no. 1, pp. 7-18, 2006.

[74] S. Peters, G. Schweibold, H. Przuntek, and T. Muller, "Loss of visual acuity under dopamine substitution therapy," NeuroOphthalmology, vol. 24, no. 1, pp. 273-277, 2000.

[75] J. T. Pearlman, A. H. Kadish, and J. C. Ramseyer, "Vision loss associated with amantadine hydrochloride use," Journal of the American Medical Association, vol. 237, no. 12, p. 1200, 1977. 


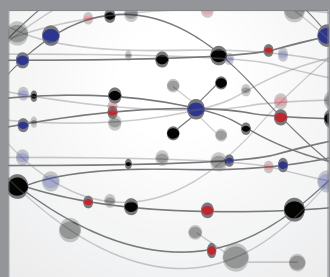

The Scientific World Journal
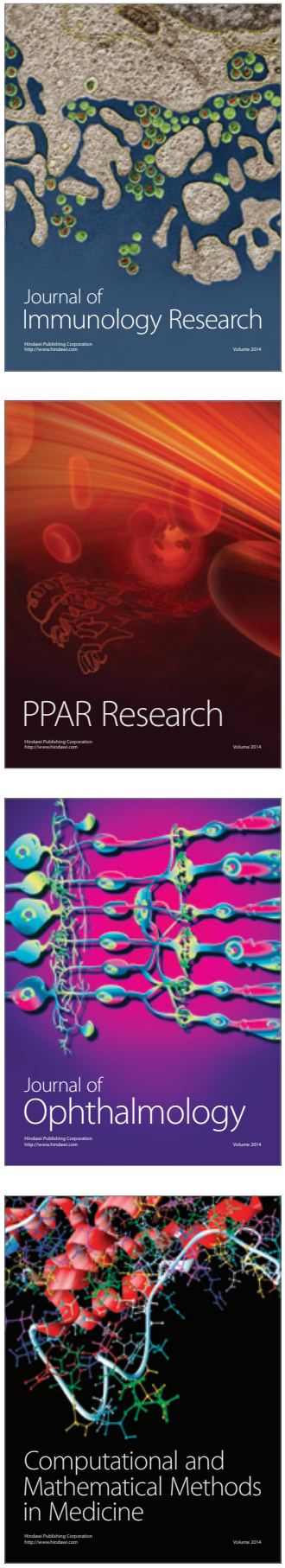

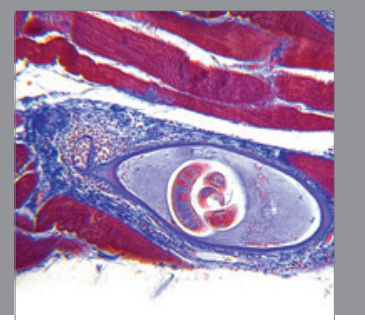

Gastroenterology

Research and Practice
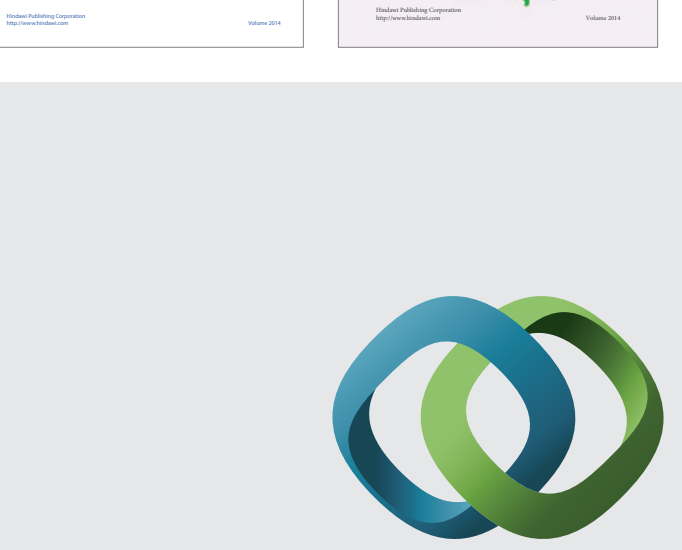

\section{Hindawi}

Submit your manuscripts at

http://www.hindawi.com
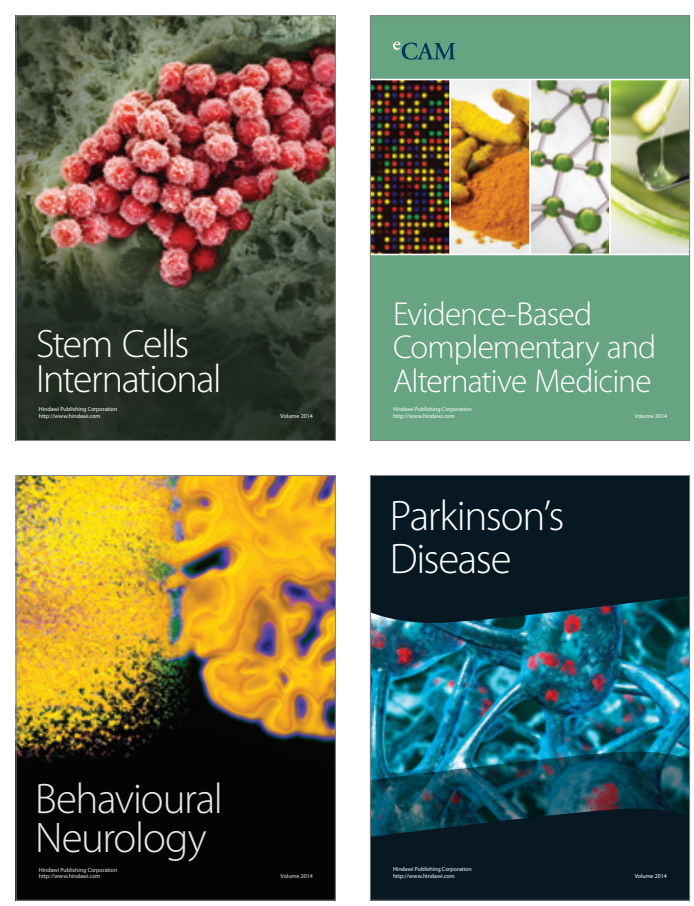

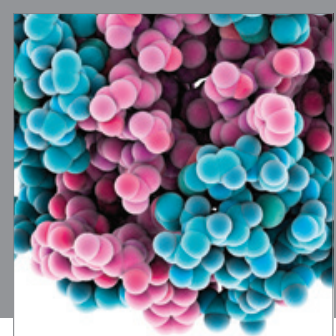

Journal of
Diabetes Research

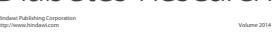

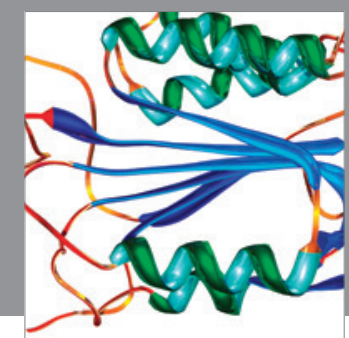

Disease Markers
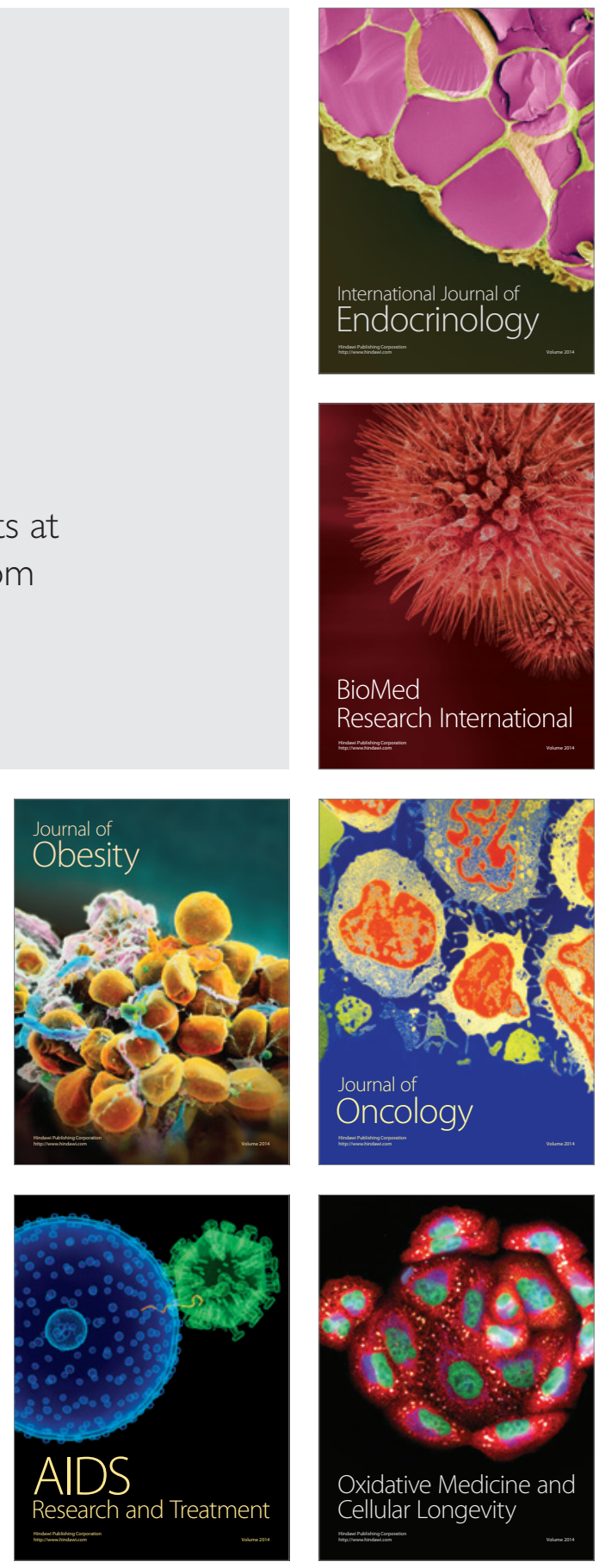